\title{
MASS LOSS FROM EARLY-TYPE STARS
}

\author{
J. B. HUTCHINGS
}

Dominion Astrophysical Observatory, Victoria, B.C., Canada

\begin{abstract}
Following the detailed study of four very high luminosity OB stars, a survey has been made for spectroscopic evidence of mass loss in a number of early-type supergiants. A list of spectroscopic criteria is given and the mass loss estimates for 24 stars plotted on the HR diagram. The dependence of the phenomenon on spectral type and luminosity is discussed as well as its significance in terms of stellar evolution.
\end{abstract}

Since Feast has mentioned the results of the analysis of three very luminous early-type stars showing evidence for extended accelerating envelopes and since the details have been published (Hutchings, 1968), I shall not go over this work again, but rather report on a less detailed survey of high luminosity early-type stars in the northern sky, to try to find how general the phenomenon of mass loss is from such stars. As a background to this discussion I shall just say very briefly that the earlier work was based on a detailed analysis of individual line velocities and profiles which was used in conjunction with theoretical work (Wellman, 1951; Menzel, 1937) on the behaviour of $\mathrm{He} \mathrm{I}$ and $\mathrm{H}$ lines in moderate radiation dilution. It was possible to relate certain lines (and thus expansion velocities) with radiation dilution ranges and hence build up a crude picture of an expanding envelope. This is used as the starting-point for an iterative method of computing strong line profiles, which converges to a structure of

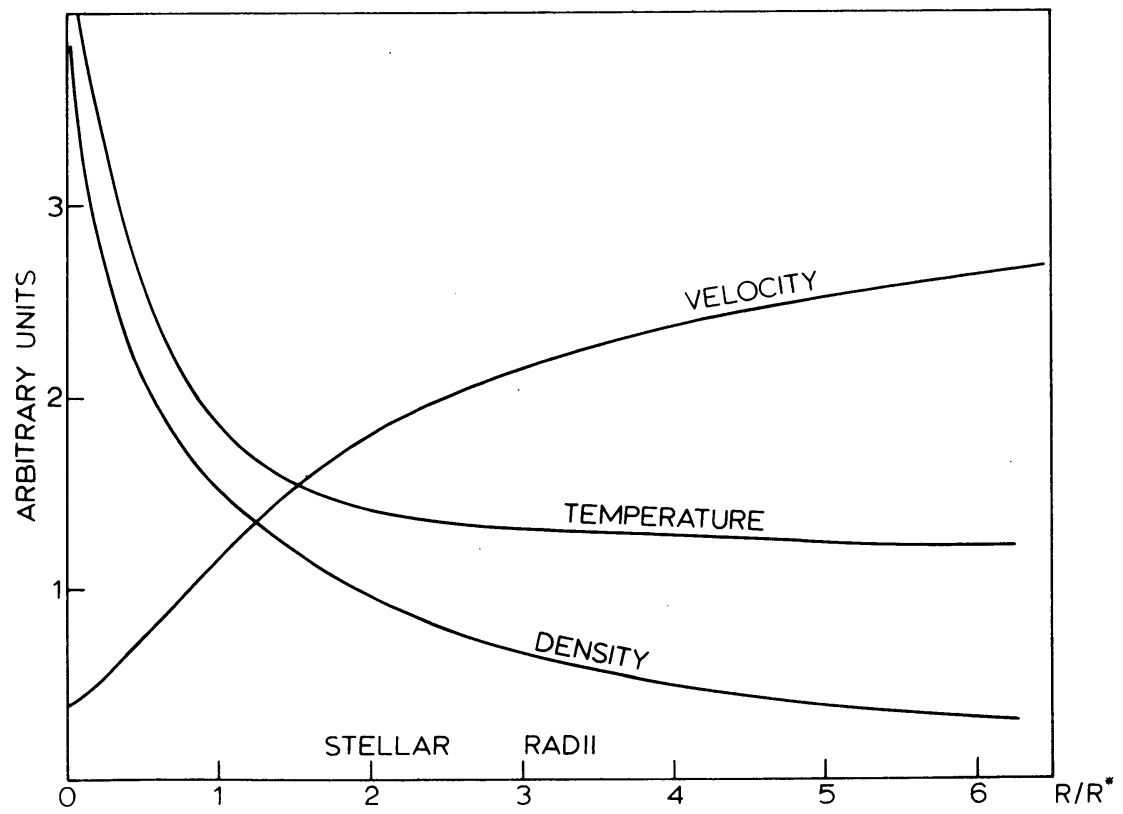

Fig. 1. Typical structure of OB supergiant outer atmosphere. 
which a typical example is shown in Figure 1. All the stars show this structure with wide variation in the maximum velocity, density and extent of the envelope. Mass loss rates for stars of spectral type about B0 and $M_{v} \sim-7.5$ are about $10^{-5} M_{\odot} \mathrm{yr}^{-1}$. In addition to the normal spectrographic evidence used in these investigations we must bear in mind the now well-known observations of the ultraviolet resonance lines which indicate velocities of expansion of some thousands of $\mathrm{km} \mathrm{sec}^{-1}$ in stars whose mass loss rates are much lower than those referred to above. We will discuss the ultraviolet lines in more detail in the next session.

\section{TABLE I}

Expanding atmosphere criteria

(1) $\mathrm{H} \alpha$ emission present. (UV resonance line shift and emission?)

(2) $\mathrm{H} \beta$ emission present, HeI $5875 \AA ̊$ emission present.

(3) Balmer velocity progression.

(4) Velocity-excitation relation present.

(5) HeI $4471 \AA, M_{\text {II }} 4481 \AA$, HeI $4026 \AA$, CII $4267 \AA$ velocities separate out.

(6) HeI $3888 \AA$ separates from $\mathrm{H} 8$.

(7) $\mathrm{H} \gamma$ emission, other HeI emission, further emission lines.

TABLE II

Mass loss strength for stars studied

\begin{tabular}{rlcc} 
Star H.D. & Type & Mv & Mass loss \\
\hline & & & \\
7583 & A0Ia & -7.1 & $(3)$ \\
14134 & B3Ia & -6.8 & 3 \\
14143 & B2Ia & -7.4 & $(3)$ \\
14818 & B2Ia & -6.4 & $(3)$ \\
24398 & B1Ib & -5.8 & 0 \\
33579 & A3Ia0 & -9.1 & $(5)$ \\
36486 & O9.5II & 5.8 & $\frac{1}{2}$ \\
37128 & B0Ia & -6.6 & 1 \\
37742 & O9.5Ib & -6.2 & 1 \\
41117 & B2Ia & -7.3 & 4 \\
45314 & O9pe & -6.8 & $(4)$ \\
91316 & B1Ib & -5.9 & 0 \\
151804 & O9f & -7.2 & 6 \\
152236 & B1Ia 0 & -8.0 & 7 \\
152408 & O8f & -7.1 & 7 \\
164353 & B5Ib & -5.2 & 0 \\
169454 & B1Ia ${ }^{+}$ & -7.7 & 5 \\
183143 & B7Ia & -7.5 & 2 \\
188001 & O8f & -6.5 & 4 \\
190603 & B1.5Ia & -7.5 & 6 \\
193237 & B1p & -8.4 & $7{ }^{+}$ \\
198478 & B3Ia & -7.2 & 2 \\
210839 & O6p & -6.8 & $(2)$ \\
223385 & A3Ia & -8.6 & $(3)$ \\
& & & \\
\hline
\end{tabular}


From the earlier investigations and the present one it became evident that there is an increase in types of spectroscopic evidence for mass loss of this nature as the phenomenon becomes more marked, i.e. as the density and expansion velocity of the envelope increase. Thus it is possible to compile a list of observable effects in approximate order, as shown in Table $\mathbf{I}$.

The phenomena 3-5 do not necessarily appear in the order given as they depend on a number of factors in the atmospheric structure. However, a good guide to the strength of mass loss is simply the number of these criteria which show up in any given star after a careful analysis has been made of several plates.

Before mentioning specific results it should also be noted that the stars showing these effects all appear to be time variable so that criteria vary in strength, and velocities change irregularly. It is thus desirable to have several well spaced observations of each star.

A survey was made on plates of dispersion $15 \AA \mathrm{mm}^{-1}$ or better on stars selected for high luminosity and early type, and detailed measurements revealed mass loss evidence in many of them.

A careful assessment was made of the $M_{v}$ of each star, using $\mathrm{H} \alpha$ calibration (Andrews, 1968), H $\gamma$ (Petrie, 1965; Walker and Hodge, 1968), H $\gamma$ (Hutchings, 1966; Kopylov, 1958) and SilII (Hutchings, 1967), and Table II shows the results for all the stars.

Figure 2 shows a plot on the H-R diagram of these stars. It is evident that the mass

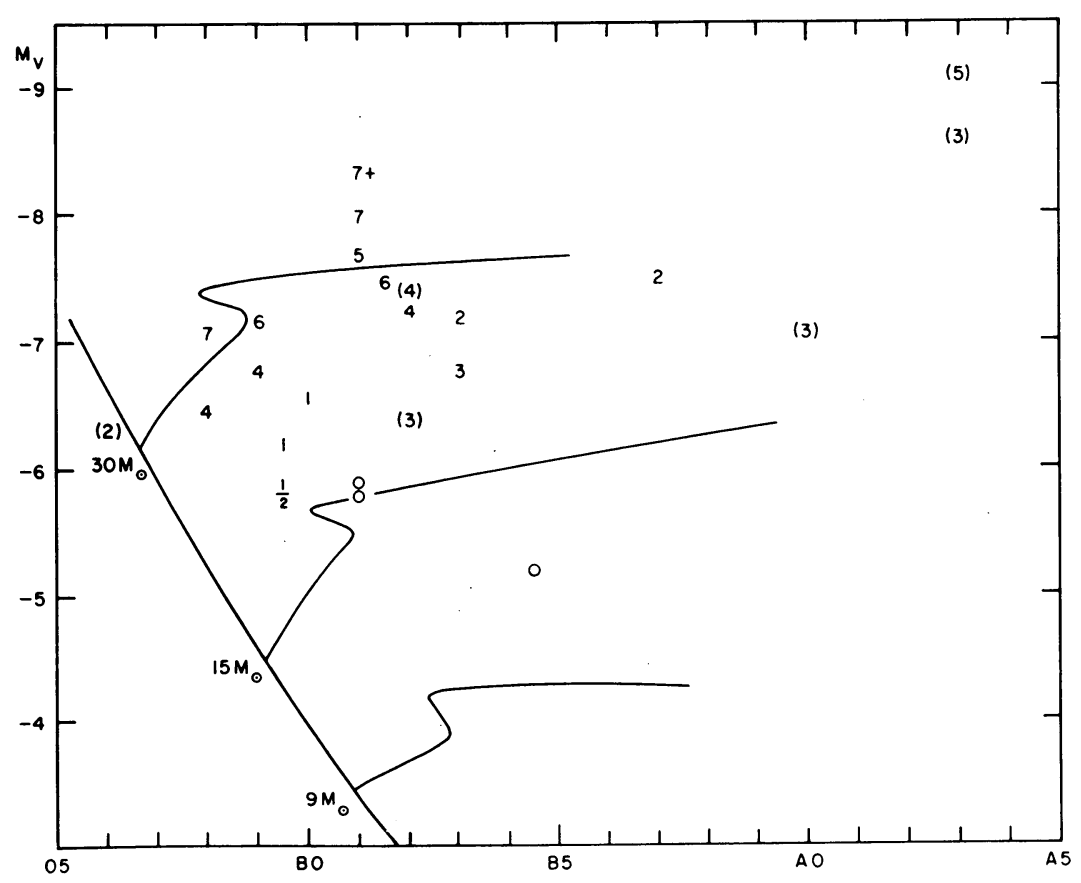

Fig. 2. H-R diagram showing mass loss criteria. 
loss depends on both luminosity and spectral type among the OB stars. The bracketed figures are those derived from less reliable plates, or the A stars, where extrapolation of the interpretative arguments for $O B$ stars may be questionable.

The detailed models made so far indicate a mass loss rate of $10^{-5} M_{\odot} \mathrm{yr}^{-1}$ or greater for stars of some $30 M_{\odot}$, and $10^{-7}-10^{-6} M_{\odot} \mathrm{yr}^{-1}$ for $15 M_{\odot}$ stars. No detailed work has yet been done on A stars and this is now being undertaken. It is evident that the mass lost may be a large portion of the total for high mass stars and therefore the problem has important stellar evolutionary consequences. In particular it is important to know whether mass loss begins before hydrogen core burning ends, as this may affect the stars' evolutionary track markedly (Hartwick, 1967). It is also important to see whether the ultraviolet data increase in strength as strikingly as the visual, as this may provide evidence on the mass loss processes. Such scans have been made with the present Orbiting Astronomical Observatory.

\section{References}

Andrews, P. J.: 1968, Mem. Roy. Astron. Soc. 72, 35.

Hartwick, F. D. A.: 1967, Astrophys. J. 150, 953.

Hutchings, J. B.: 1966, Monthly Notices Roy. Astron. Soc. 132, 433.

Hutchings, J. B.: 1967, Thesis, Cambridge University.

Hutchings, J. B.: 1968, Monthly Notices Roy. Astron. Soc. 141, 219 and 329.

Kopylov, I. M.: 1958, Izv. Krymsk. Astrofiz. Observ. 20, 156.

Menzel, D. H.: 1937, Astrophys. J. 85, 330.

Petrie, R. M.: 1965, Publ. Dominion Astrophys. Obs. 12, 317.

Walker, G. A. H. and Hodge, S. M.: 1968, Publ. Astron. Soc. Pacific, 80, 290.

Wellman, P.: 1951, Z. Astrophys. 30, 71 and 88.

\section{Discussion}

Conti: Only one star in your diagram of 'Mass loss' is near the main sequence; which star is this? Hutchings: It is an O6 star, HD 210839, for which I have slightly inferior data at present.

Peytremann: Which is the mechanism responsible for the mass loss? Is it radiation pressure or rotation of the star?

Hutchings: As discussions in the next session should show, it looks like radiation pressure. The detailed analysis I have done on a few stars indicate a spherically symmetrical envelope and a rotational velocity of $80 \mathrm{~km} / \mathrm{sec}$ or less. There is no indication of the type of profile found in the equatorially extended atmospheres around fast rotating main sequence Be stars. 